\title{
Heroin injecting and the introduction of HIV/AIDS into a Scottish city
}

\author{
Roy Robertson ${ }^{1} \quad$ Alison Richardson ${ }^{2}$
}

J R Soc Med 2007; 100:491-494

Although several accounts of the introduction of HIV infection into the drug-using population in the UK have been attempted by various contributors, the complete story remains somewhat opaque and surrounded in mystery, perhaps all the more interesting and intriguing for these reasons. Although this paper is unlikely to be the end of the story or even to present a totally clear revelation, it is worth adding some information in an attempt to increase the likelihood of us all learning from an unfortunate medical and public health disaster. Medical stories are common in the history of Edinburgh and infectious diseases contribute a significant number, including plague, cholera and tuberculosis, but the interest in HIV should go into history as being at least as important as these others.

The history of opiate use is long and its origins are lost in antiquity; in many countries where opium is cultivated, it has been the prevailing addictive drug and used by a significant sector of the population in the same way that alcohol is a problem in western countries. A large section of the population in Victorian England used opioid-containing linctuses, and many were addicted. Literature is full of examples of opium use, and Coleridge famously claimed that his fantastical poem Kubla Khan was inspired by an opium dream. Inappropriate heroin use is recorded after its synthesis in the 19th century and the phenomenon of illegal injecting emerged after the invention of the reusable hypodermic needle and syringe. It was not, however, until comparatively recent times that the practice of heroin injecting became common. Case studies of hepatitis B in groups of injecting drug users reported in the 1970s created interest for a minority of medical specialists only. None of this prepared us for the emergence of the heroin use of the 1980s and beyond, which became commonplace in countries and cities with no prior experience of intravenous drug use.

\footnotetext{
${ }^{1}$ General Practitioner, Muirhouse Medical Group, 1 Muirhouse Avenue, Edinburgh EH4 4PL, UK; and Reader, Department of Community Health Sciences, Edinburgh University

${ }^{2}$ Consultant Clinical Psychologist, Spittal Street Centre, 22-24 Spittal Street, Edinburgh EH3 9DU, UK

Correspondence to: Dr Roy Robertson

Email: Roy.Robertson@ed.ac.uk
}

At some point during the period 1980-1983 a strange thing happened in Edinburgh as a result of a number of disparate factors coinciding. Although the phenomenon of increasing use of heroin by young people had been observed in several UK cities, Edinburgh was experiencing events which would later identify the drug scene there as distinctly different from that in the rest of the UK-quite unique, in fact.

The emerging crisis came in the form of increasing supplies of heroin, reputedly from Afghanistan and Iran in the wake of political upheaval in these countries. Excessive supplies in many European cities were evident and a shift of consumer from the student or dissident class to the socially deprived populations in inner-city housing estates gave a new profile to the problem. Medical services described damage done by injecting. Cases of hepatitis, abscess and endocarditis became common. Forensic pathologists noted a rise in sudden deaths in young people. Perhaps, however, the profession with the most involvement was the criminal justice system, which saw increasing numbers of individuals accused of possessing heroin either for personal use or for supplying to others being brought before the courts.

As ever, and in retrospect, it is easy to see that there was a social change happening which was neglected and in some sectors ignored completely until much damage had been done. In 1980, however, a confident Government was uninterested in a compassionate response to a problem viewed as one of lawlessness and social disorder rather than a symptom of social malaise or a medical and public health emergency. The response was aggressive and authoritarian and completely missed the important warning signs that a humanitarian and medical crisis was emerging.

By 1983, there was still no public health response or coordinated strategy across the caring and law enforcement agencies. A document published in 1982 by the Advisory Council on the Misuse of Drugs explained the role of social work and rehabilitation but made little or no reference to health, and the 1984 Guidelines on the Treatment of Drug Misuse issued by the four UK Departments of Health considered that medically supervised detoxification was a simple and short-term process with spontaneous remission possible after a short two-week course of methadone. A practical and pragmatic response from a surgical supplies retailer in Edinburgh city centre was to sell, at low cost, 
needles and injecting equipment even in the knowledge that these would be used to administer illegal drugs. In September 1982, due in part to the prevailing political climate and resulting pressure from local pharmacies and general practitioners (GPs), the shop was closed. The ability of drug users in Edinburgh to obtain clean injecting equipment was consequently even more severely curtailed. Worse was to follow.

In 1984 the police and courts in Scotland's main cities were taking a new interest in drug misuse cases. This was backed by strong support from the Solicitor General and Lord Advocate. Cases were increasingly referred for High Court trial by judge and jury, rather than for Summary trial where the penalties are limited. Lawyers and advocates reported individuals who were given long custodial sentences for small quantities of heroin which was clearly, in retrospect, for their own use rather than for sale to others for profit.

The numbers of heroin users increased. The heavyhanded response of the police, courts and the medical establishment, in their interpretation of the law and other guidance, served only to send injecting drug use further underground.

A report from Glasgow by the non-statutory drug organization SCODA (Standing Conference on Drug Abuse) in 1982 identified a rapid increase in heroin use in Edinburgh. A small research project was funded in Muirhouse Surgery to support an investigation into the cases seen in that practice and the suggestion that injecting was expanding and that hepatitis cases were increasing. Blood samples were taken and tested for hepatitis to assess the extent of the problem. Sporadic meetings were being organized to discuss hepatitis prevention and in that practice, but few others, a philosophy of damage limitation was being pursued, which included provision of injecting equipment for patients who were unwilling or unable to cease injecting.

Cases continued to increase, made worse for GPs because of the closure of the city's only methadone clinic at the Royal Edinburgh Hospital. Methadone was out of favour with officialdom and alternatives were frowned upon. Efforts were still concentrated on short-term detoxification and achieving abstinence even in the face of intractable use and damage.

Across the Atlantic in the USA a separate story was emerging. The identification of the acquired immunodeficiency syndrome (AIDS) among homosexual men in California and New York was causing social change and political stress. Social change, in the form of liberalization of the homosexual community, and the political response to the emergence of bath houses where men could have sex with each other, contributed to the disease. Randy Shilts, in his amazing account of the early years of the US epidemic, described the devastation and suffering experienced and the complex interaction between social groups within the USA and further afield. He explored the possible lines of transmission within the homosexual community and from drug injectors, those infected from blood or blood products and heterosexuals. Drug injectors were seen as a secondary group but one with the potential to transmit the virus rapidly and to disseminate it into the non-drug-using heterosexual population. This made politicians more interested. If minority groups such as homosexuals, drug users and the unfortunate recipients of infected blood were at risk it was one thing, but to contemplate the wide dissemination into the population raised the spectre of an African-style epidemic potentially affecting everyone. In 1984 there was awareness of the problems in Uganda and Zaire, but South Africa, Zimbabwe and Botswana had still not declared a problem and tests to identify those incubating the disease were still not available.

This lack of ability to identify the organism responsible for the clinical condition characterized by wasting, multiple infections and a slow debilitating deterioration meant that patients and doctors had no way of predicting who was infected before illness ensued, and there was little understanding of the length of time from infection to illness. The belief that the incubation period might be 12 or 18 months gave rise to a serious underestimate of the nature of the crisis and the seriousness of the global situation. In addition there was, until 1985, no sure way of knowing how infectious the organism was and whether or not casual, non-sexual contacts such as household contacts were at risk. The ability to test for an antibody in the blood was to bring both increased anxiety and reassurance.

In 1985 a test became available for the detection of antibodies to the human immunodeficiency virus (HIV), then known as HTLV-3, and the world changed. Medicine became involved with politics, commerce and the media. Public health became a major participant in planning, prevention and social change. Doctors in specialties such as sexually transmitted diseases and infectious diseases became celebrities, and scientists — previously confined to laboratories and experimental journals - emerged as businessmen with an economic interest in health. As might be expected, the political establishment and the media were, for the time being, the driving force.

In Edinburgh the test was applied to the local problem of most interest, the injecting-drug-user population. Virologists and a local public health doctor retrieved some samples from patients attending the accident and emergency department who were known to be injecting drug users and tested blood for the antibody to HIV. Over $60 \%$ of those tested were positive. This was recognized to be important, but just how important was difficult to assess. At the time the incubation period of 
HIV was still thought to be 12 to 18 months from infection to the onset of AIDS.

Elsewhere, antibody tests were applied to risk groups and to laboratory stores of blood samples. A study in New York of household contacts showed no positives in individuals who were not otherwise at risk. This was reassuring. The test, however, revealed large numbers of individuals who were infected but not yet ill. The incubation period question raised a new spectre. Perhaps the incubation period was several years (it transpired later that there was great variability, with some becoming ill within a short period of time and others remaining asymptomatic for up to 20 years). A long incubation period would mean that people had been infected much earlier than they may have thought and that the spread was likely to be greater and more widespread.

The African situation was still unclear in 1985. Symptomatic 'Slim disease' was well known in Uganda and some other central African countries, but in countries such as South Africa and Zimbabwe no testing had been done in poorer and rural communities and cases were not yet evident. Most of the world-wide focus was still on homosexual men in the USA. Drug users were seen as a high-risk group but outside New York, even in California, cases were few and did not merit big headlines or serious political concern.

A conference in 1986 in Newark, New Jersey, changed this and opened eyes to the plight of drug users and the startling revelation that, being mostly heterosexual, they represented an introductory passage into the nonhomosexual, non-drug-using population. This became serious politics. The Edinburgh data presented at that meeting clearly focused the scientists present on the young, white, drug-using population and their sexual partners and children. Edinburgh was suddenly on the international map as the epicentre of a potential explosion of African-style transmission.

1986 was the year of the HIV test. Across the globe testing was applied to all sorts of groups and individuals suffering from wasting diseases, those who had had a blood transfusion or treatment with a blood product, homosexual men, current and abstinent drug users and their current and previous sexual partners and any anxious heterosexual. In fact many tests were merely for reassurance and to confirm what was already known, but within months the pattern of the epidemic was becoming clearer. There were clearly hot spots and sad stories. In Edinburgh, as well as the spread among drug injectors there were a small number of sexual partners and children infected. Most of the severe haemophiliac cohort in Edinburgh was infected as a result of contaminated Factor 8, the blood product which prevents bleeding, and some blood-transfusion recipients were also infected. Many homosexual men who had previously thought that the disease was going to pass them by were disappointed. In Europe there were similar examples in Italy and Spain of rapid spread of HIV among drug injectors, but most centres such as Amsterdam and Paris showed slow endemic patterns of spread.

In most UK cities, however, there were few if any infected drug users and attention turned to prevention rather than treatment. Resources were galvanized and widely distributed in an attempt to put in place measures, many untested, to prevent the spread of HIV among injectors. Many measures were considered and discussed. These varied from isolating those infected to widespread distribution of injecting equipment and even the drugs themselves. Diversions from drugs into employment, residential centres, religious communities and medical treatment centres all gained support. Cases were becoming more common. The incubation period between infection with the HIV virus and the development of AIDS was still unclear, as was the information about the timing of the initial spread in Edinburgh, but cases were emerging and young patients were being seen in surgeries and hospitals in Edinburgh with the complex symptoms often present when the body's immune system begins to fail. Symptoms such as extreme breathlessness caused by the infective agent Pneumocystis pneumoniae, diarrhoea caused by bowel infections, dementing illness, severe weight loss and unusual malignancies were becoming more common. In retrospect, earlier sudden deaths were attributed to clandestine infection with HIV.

By this time, 1987, the World Health Organization had been notified of 43,880 cases of AIDS in 91 countries, and reactions ranged from national and international searches by governments and health agencies for explanations and solutions to personal panic and terror. Many local and national agencies seemed to be paralyzed with indecision, unable to reconcile the new agendas and revelations with traditional values and conservative intuitions. It was as if a cataclysmic event had set a need for a new approach to personal behaviour - sexual liberty versus public health and public health protection in an age of travel and technology initiating change at a pace not seen since the industrialization of the western world.

It was in 1987 that the famous UK campaigns were launched involving TV adverts which showed 'the tip of the iceberg' and tombstone images. Leaflets delivered to every household proclaimed 'AIDS. Don't die of ignorance. Anyone can get it, gay or straight, male or female. Already 30,000 people are infected'. The major influence on this was the fear that the infection would spread into the heterosexual community as a result of sexual contact with drug users. There was much less regard for the hapless human beings who were already infected. There was little information about what might happen to them and estimates 
of illness and mortality were guesstimates, based on widely differing reports from around the world.

Important in the development of a response to the AIDS problems in Edinburgh and the driving force behind the epidemic, the injecting-drug-use culture, was the treatment service for heroin-addicted patients. This changed slowly between 1980 and 1987, being largely located in general practice and the residual psychiatric drug dependency unit. In 1987 new services were established. The public health medicine consultant at the time pioneered both the establishment of a Needle Exchange Service and of a Drug Problem Service whose ethos was based on harm reduction. The services developed at a time of recognition of the urgent need to divert a bigger epidemic, and were based on the assumption that the risk of giving clean needles and syringes was less than that of allowing reckless sharing to continue. Both initiatives have developed beyond the initial conception to provide ongoing maintenance treatment and easy access to injecting equipment for anyone involved in illegal drug injecting. Similar services have developed in many western countries and most UK cities and proposed extensions continue to be necessary.

The present, rather chilling, normality of drug use and drug-related deaths among young people and the apparently relentless spread of injecting drug use in many countries could not have been predicted at the time of the emergence of HIV, when it was expected that such a crisis might make injecting redundant as a pastime. Where HIV infection has gone among injecting drug users and their sexual partners is similarly mysterious. Most new transmissions in the UK are among other high-risk groups, most commonly homosexual men or heterosexuals who have been infected in subSaharan Africa, but drug injectors still account for some new cases and another epidemic seems quite possible. Predicting the future is of course shrouded in uncertainty. The spread of another virus, that causing hepatitis $\mathrm{C}$, is an indicator that vigilance is the essential part of prevention and that expenditure on prevention, education and opportunity are as important as technology in preventing future similar public health disasters. 